$11-15-2008$

\title{
Micromechanics Model Based on the Nanostructure of PFSA Membranes
}

\author{
Ahmet Kusoglu \\ University of Delaware \\ Michael H. Santare \\ University of Delaware
}

Anette M. Karlsson

Cleveland State University, a.karlsson@csuohio.edu

William B. Johnson

Gore Fuel Cell Technologies

Simon Cleghorn

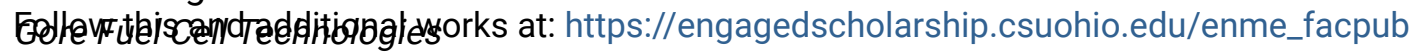

Part of the Mechanical Engineering Commons, and the Membrane Science Commons How does access to this work benefit you? Let us know!

\section{Publisher's Statement}

This is the accepted version of the following article: Kusoglu, A., Santare, M. H., Karlsson, A. M., 2008, "Micromechanics Model Based on the Nanostructure of PFSA Membranes," Journal of Polymer Science Part B: Polymer Physics, 46(22) pp. 2404-2417., which has been published in final form at http://onlinelibrary.wiley.com/doi/10.1002/polb.21573/abstract

\section{Original Citation}

Kusoglu, A., Santare, M. H., Karlsson, A. M., 2008, "Micromechanics Model Based on the Nanostructure of PFSA Membranes," Journal of Polymer Science Part B: Polymer Physics, 46(22) pp. 2404-2417.

This Article is brought to you for free and open access by the Mechanical Engineering Department at EngagedScholarship@CSU. It has been accepted for inclusion in Mechanical Engineering Faculty Publications by an authorized administrator of EngagedScholarship@CSU. For more information, please contact library.es@csuohio.edu. 


\title{
Micromechanics Model Based on the Nanostructure of PFSA Membranes
}

\author{
AHMET KUSOGLU, ${ }^{1}$ MICHAEL H. SANTARE, ${ }^{1}$ ANETTE M. KARLSSON, ${ }^{1}$ SIMON CLEGHORN, ${ }^{2}$ \\ WILLIAM B. JOHNSON ${ }^{2}$ \\ 'Department of Mechanical Engineering, University of Delaware, Newark, Delaware 19716 \\ ${ }^{2}$ Gore Fuel Cell Technologies, 201 Airport Road, Elkton, Maryland 21922
}

\section{INTRODUCTION}

Perfluorosulfonic acid (PFSA) polymer is used in proton exchange membranes (PEM) for fuel cell applications due to its inherent electrochemical properties. PFSA membranes are a class of fluoropolymers consisting of a hydrophobic polytetrafluoroethylene (PTFE) backbone attached to hydrophilic sulfonic acid groups $\left(\mathrm{SO}_{3}{ }^{-}\right)$via fluorocarbon polymer side chains (Fig. 1). Upon water edu)

Correspondence to: M. H. Santare (E-mail: santare@udel. uptake, these sulfonic acid groups ionize and attach to the water molecules to provide a conductive path for proton transport, whereas the polymer network maintains the overall structure of the membrane. However, various failure mechanisms in the form of chemical degradation and mechanical damage are observed during fuel cell operation, imposing a crucial technical barrier to the widespread commercialization of PEM fuel cells. ${ }^{1-3}$ Mechanical stresses generated in the membrane during hydration-dehydration cycling initiate and propagate this damage: during the operation of the cell, inelastic compressive stresses are induced in the membrane upon hydration leading to residual tensile stresses 


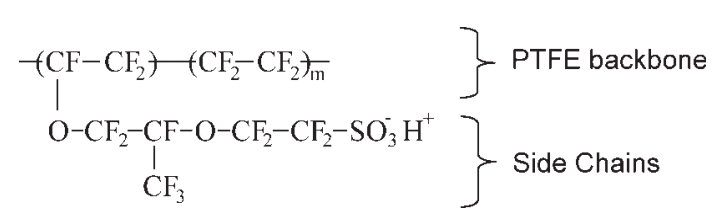

Figure 1. Chemical structure of a PFSA membrane.

during dehydration. Our previous studies ${ }^{4-6}$ showed that these stresses are affected by the temperature and moisture-dependent mechanical properties of the membrane. Thus, establishing the mechanical properties and their dependence on swelling (or water content) and temperature is important for the characterization of the mechanical behavior of PFSA membranes. This is the focus of our study. In the following section, studies in the literature on the nanostructure of PFSA membranes are reviewed to establish the basis for our mechanics model.

\section{Nanostructure of PSFA Membranes}

Early studies on the morphology of PFSA membranes $^{7,8}$ suggested that the nanostructure of PFSA membranes can be characterized by a cluster-network model, where the ionic sulfonic acid groups $\left(\mathrm{SO}_{3}{ }^{-}\right.$groups) aggregate into domains forming inverted spherical micellar structures (the so-called clusters) dispersed throughout the perfluorinated matrix [Fig. 2(A)]. Upon hydration, water molecules initially attach to the $\mathrm{SO}_{3}{ }^{-}$ groups by ionizing them. Additional water molecules increase the size of these clusters, which results in macroscopic swelling of polymer. In this work, we consider the cluster to be the spherical domain where both $\mathrm{SO}_{3}{ }^{-}$groups and water molecules are located. When a critical volume density of water is reached, the clusters make connections through the matrix and form a conductive pathway for $\mathrm{H}^{+}$ions and the diffusion of water molecules across the membrane (percolation) [Fig. 2(B).$^{8-10}$ This water-cluster network is embedded in the perfluorinated matrix with the $\left(\mathrm{SO}_{3}{ }^{-}\right)$ionic groups located at the polymer-water interface. ${ }^{7,8}$ It is suggested that this structure can be represented by spherical clusters of diameter $d_{\mathrm{s}}$ in a cubic lattice of length $d$ connected by cylindrical channels [Fig. 2(D)]. ${ }^{7,8,11}$ In the absence of water, the clusters still exist, due to the tendency of $\mathrm{SO}_{3}{ }^{-}$ionic groups to aggregate to minimize the free energy [Fig. 2(C)]. ${ }^{12}$ Thus, water uptake results in an increase in the diameter of the cluster and the length of the cubic lattice from their respective initial values, $d_{\mathrm{s}}^{\text {dry }}$ and $d^{\text {dry }}$ [Fig. 2(C,D)]. During water absorption, coalescence of clusters causes a decrease in the number of clusters as the clusters grow, leading to a continuous structural reorganization. ${ }^{7,8,10,13-15}$

A phase-separated nanostructure for swollen PFSA membranes with an average cluster diameter of between 3 and $6 \mathrm{~nm}$ has been validated experimentally through several methods, for example, small- and wide-angle scattering with neutrons (SANS) and X-Rays (SAXS). ${ }^{10,13-23}$ Modifications for the afore-described cluster-network model and other nanostructural representations have been proposed as described elsewhere. ${ }^{13,18,24-26}$ (For a review and comparison of these models see Mauritz and Moore ${ }^{27}$ and Schmidt-Rohr and Chen. ${ }^{28}$ )

Even though the literature lacks a clear consensus on the size and shape of channels, a continuous hydrophilic phase similar to a porous structure is required to explain the water transport mechanisms and ion conductivity in PFSA membranes. ${ }^{8,13,14,16,18,27,29,30} \mathrm{Hsu}$ and Gierke calculated the diameter of the channels connecting the spherical clusters as 1-2 nm. Also, several authors have investigated the size distribution of the pores in swollen PFSA membranes to characterize the nanostructure and to understand the transport properties. By assuming a cylindrical shape for all pores, Koter" ${ }^{31}$ determined an "equivalent pore radius" from the electroosmotic transport of water and reported a value of 1.1-1.2 nm for Nafion ${ }^{\circledR} 117$ membranes. (Nafion membrane is a commercially available PFSA-based membrane commonly used in PEMFC applications. Nafion is a registered trademark of E.I. DuPont De Nemours \& Co.) Using standard porosimetry, Divisek et al. $^{32}$ determined the volume and the surface area distribution of pores as functions of the pore size. They reported an average pore radius of 1-2 $\mathrm{nm}$, independent of temperature for Nafion membranes using this method. Iijima et al. ${ }^{20}$ used differential scanning calorimeter to determine the cluster size distribution in PFSA membranes and calculated an average diameter of between 1 and $2.5 \mathrm{~nm}$ for the cylindrical channels connecting the spherical clusters.

In addition to the size and shape of the clusters, Dreyfus ${ }^{24,33}$ studied the spatial arrangement and proposed a structure that minimizes the distance between first neighbors; a diamond (tetrahedron) model, where each cluster has four nearest neighboring clusters located at a well-defined distance, suggesting a strong local order. ${ }^{15}$ 
Dreyfus $^{33}$ also suggested that each cluster can be represented by a sphere connected to its neighbors by four cylindrical channels. He used this assumed configuration to confirm the calculated percolation threshold measured experimentally. Later, it was shown that this local order model can be used to characterize the spatial distribution of swollen clusters in PFSA membranes as observed in SANS experiments for water volume fractions up to $0.3-0.4{ }^{13,15,17}$

Recently, Schmidt-Rohr and $\mathrm{Chen}^{28}$ reviewed the existing nanoscale representations for the PFSA membrane and suggested that water is confined in parallel-cylindrical channels of 1.8-3.5 $\mathrm{nm}$ in diameter, which are distributed in polymer backbone with the hydrophilic side-chains at the interface.

\section{Outline and Motivation}

The main goal of this study is to develop a micromechanics-based model that can be used to predict Young's modulus of PFSA membranes at various water contents and temperatures. In our micromechanics approach, we will use the idealizations of the nanostructure discussed earlier and neglect any energetic interactions among the components. First, the relationship between the relative humidity, $\mathrm{RH}$, and the water content, $\lambda$, is described using the formulations available in the literature for the interpretation of experimental data. Then, micromechanics models are developed for a representative volume element (RVE) inspired by the existing literature on the nanostructure of PFSA membranes, reviewed earlier. The resulting Young's moduli are compared with previously published experimental data ${ }^{34}$ over a range temperatures and with other empirical models of PFSA membranes. For simplicity, in this study we assume (i) homogeneous cluster size distribution, where all clusters swell equally, an idealization used in several models as described elsewhere ${ }^{7,24,29}$ and (ii) the shape of the interconnecting channels is cylindrical as in the models proposed in other studies. $8,13,20,29,30$

\section{SORPTION BEHAVIOR OF PFSA MEMBRANES}

Nafion membranes are classified by their thickness and equivalent weight, $\mathrm{EW}(\mathrm{g} / \mathrm{mol})$, which is the number of grams of dry polymer per mole of $\mathrm{SO}_{3}{ }^{-}$group. Therefore, for a given mass of dry membrane, the molar content of sulfonic acid groups can be calculated as follows:

$$
\operatorname{mol}\left(\mathrm{SO}_{3}^{-}\right)=\frac{m_{\mathrm{p}}}{\mathrm{EW}}=\frac{V_{\mathrm{p}} \rho_{\mathrm{p}}}{\mathrm{EW}},
$$

where $\mathrm{m}_{\mathrm{p}}, \rho_{\mathrm{p}}$, and $V_{\mathrm{p}}$ are mass, density, and volume of the polymer, respectively. In this study, we will focus on PFSA membranes with $1100 \mathrm{EW}$, for which we have experimental data. ${ }^{34}$

The water content in the membrane, $\lambda$, is the number of water molecules per $\mathrm{SO}_{3}{ }^{-}$group. Using the definition of $\mathrm{EW}, \lambda$ can be written in the form:

$$
\lambda=\frac{\operatorname{mol}\left(\mathrm{H}_{2} \mathrm{O}\right)}{\operatorname{mol}\left(\mathrm{SO}_{3}^{-}\right)}=\frac{\Delta m_{\mathrm{w}}}{m_{\mathrm{p}}} \frac{\mathrm{EW}}{18}=\frac{\Delta V_{\mathrm{w}}}{V_{\mathrm{p}}} \frac{\rho_{\mathrm{w}}}{\rho_{\mathrm{p}}} \frac{\mathrm{EW}}{18},
$$

where $\Delta m_{\mathrm{w}}=m_{\mathrm{p}}^{\text {swollen }}-m_{\mathrm{p}}^{\text {dry }}(\mathrm{g})$ is the mass gain of the membrane with respect to the dry state due to the water uptake, $18 \mathrm{~cm}^{3} / \mathrm{mol}$ is the molar volume of water, and $\rho_{\mathrm{w}}\left(\mathrm{g} / \mathrm{cm}^{3}\right)$ is the density of water. $\Delta V_{\mathrm{w}}$ is the volume change with respect to the initial volume, $V_{\mathrm{p}}$, which is the volume of dry polymer. One can convert the water mass gain into the volume fraction of water by assuming that the water uptake is directly associated with the growth of the hydrophilic regions, as the polymer backbone is hydrophobic. The polymer volume fraction of a swollen membrane, $\phi_{\mathrm{p}}$ $\left(=1-\phi_{\mathrm{w}}\right)$, can then be determined directly from eq 2 as

$$
\left.\phi_{\mathrm{p}}=\frac{V_{\mathrm{p}}}{V_{\mathrm{p}}+\Delta V_{\mathrm{w}}}=1+\lambda \frac{18}{\mathrm{EW} / \rho_{\mathrm{p}}}\right)^{-1} .
$$

The sorption behavior of PFSA membranes has been investigated both theoretically and experimentally in many studies. ${ }^{9,10,18,30,35-39}$ Furthermore, based on the experimental data and theoretical models describing the swelling mechanisms, the relationship between water content, $\lambda$, and the water vapor activity, $a$ (or relative humidity, RH), has been investigated. Of the several models in the literature characterizing the sorption of PFSA membranes of EW $=1100$, the one given by Thampan et al. ${ }^{37}$ is adopted here:

$$
\lambda(a)=\lambda_{\mathrm{m}} \frac{C a\left[1-\left(n_{2}+1\right) a^{n_{2}}-n_{2} a^{n_{2}+1}\right] /(1-a)}{1-(C-1) a-C a^{n_{2}+1}},
$$

where the empirical constants are $C=150 ; \lambda_{\mathrm{m}}=$ $1.8, n_{2}=\lambda_{\text {sat }}^{\text {liq }} \lambda_{\mathrm{m}}$, and $\lambda_{\text {sat }}^{\text {liq }}=22$. For the sake of 
\begin{tabular}{|l|l|l|}
\hline A & Dry State \\
\hline
\end{tabular}

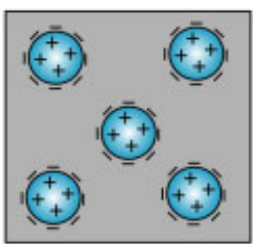

C $\mid$ Dry State

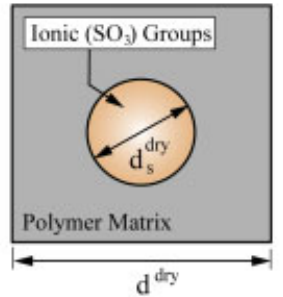

Swollen State

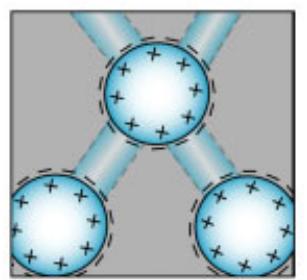

\begin{tabular}{|l|l}
\hline D & Swollen State
\end{tabular}

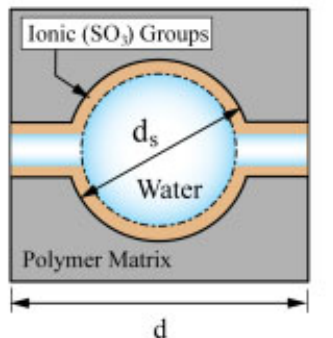

Figure 2. Cluster-network model for PFSA membranes in (A) dry and (B) swollen state and the corresponding geometric representation, consisting of a spherical cluster in a polymer matrix, shown in twodimensional view for (C) dry and (D) swollen state.

comparison, we also show another relationship given by Springer et al. ${ }^{38}$ :

$$
\lambda(a)=0.043+17.81 a-39.85 a^{2}+36.0 a^{3} .
$$

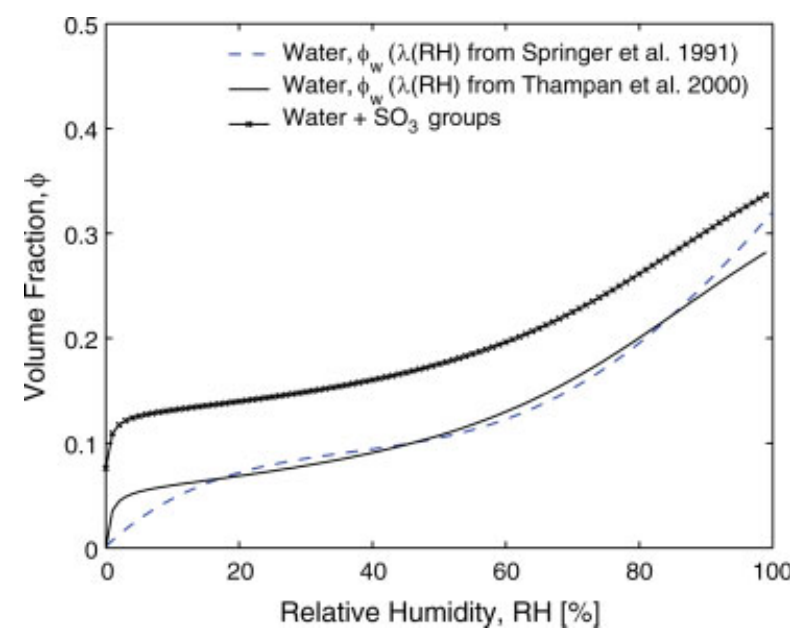

Figure 3. Evolution of the volume fractions of water and pore (consisting of sulfonic acid groups and water) as a function of relative humidity, $\mathrm{RH}$. To determine the volume fractions, two models describing $\lambda-\mathrm{RH}$ relationship are used for comparison: Springer et al. ${ }^{38}$ and Thampan et al. ${ }^{37}$ Cluster volume fraction is calculated from the water volume fraction as determined from the latter model.
Both these models can be used to obtain the sorption curve of PFSA membranes in water vapor at room temperature. However, the uptake of water molecules of PFSA membranes in liquid water is seen to increase dramatically compared with that of saturated water vapor, even though the water activity is $100 \%$ in both cases. This phenomenon is known as Schroeder's Paradox ${ }^{40}$ and discussed in several studies. 9,30,39,41 However, Onishi et al. ${ }^{39}$ showed that the water uptake is same if the membranes have the same thermal history, and the water uptake changes if the membranes are pretreated differently. In this study, we will consider the sorption from water vapor only, for which the relationship between water volume fraction and $\mathrm{RH}$ is depicted in Figure 3 using the $\lambda-\mathrm{RH}$ relationship given in eqs 4 and 5 . As seen from the figure both models give similar predictions, especially in the range of $10-95 \% \mathrm{RH}$.

The influence of temperature on the relationship between water content and relative humidity is not well established, and the literature presents contradictory information on this issue. Among the experimental studies where $\lambda$ is computed based on mass gain in the PFSA membrane at various temperatures and humidities, Zawodzinski, ${ }^{9}$ Morris and Sun $^{36}$ and Jalani et al. ${ }^{42}$ observed a slight increase in $\lambda$ with increasing temperature, especially at high humidities. However, experimental data by Hinatsu et al., ${ }^{43}$ and models developed by Weber and Newman ${ }^{30}$ and Takata et al. ${ }^{44}$ indicate that $\lambda$ decreases with

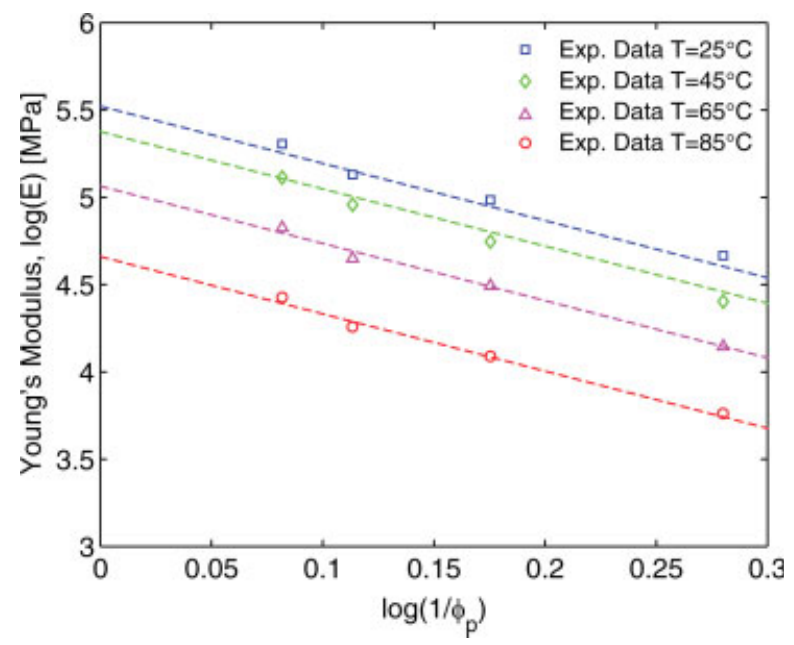

Figure 4. The log-log plot of the experimental data for Young's modulus of Nafion 112 membrane ${ }^{34}$ as a function of the reciprocal polymer volume fraction (equals to the volumetric swelling strain) depicted at four temperatures. 
Table 1. The Values of Young's Modulus of a Dry Nafion ${ }^{\circledR}$ Membrane, $E_{\text {dry }}$, Determined by the Extrapolation of the Experimental Data to Zero Water Content Given at Four Temperatures, Power-Law Exponent, $n$, and the Constants of Eq. (7) Obtained by Linear Curve-Fit to the $E_{\text {dry }}$ Values as a Function of Temperature

\begin{tabular}{|c|c|c|c|c|c|c|}
\hline \multicolumn{4}{|c|}{$E_{\text {dry }}(\mathrm{MPa})$} & \multirow[b]{2}{*}{$n$} & \multirow[b]{2}{*}{$\begin{array}{c}\kappa \\
(\mathrm{MPa} / \mathrm{K})\end{array}$} & \multirow[b]{2}{*}{$\begin{array}{c}E_{\text {dry }}^{0} \\
(\mathrm{MPa})\end{array}$} \\
\hline$T=25^{\circ} \mathrm{C}$ & $T=45^{\circ} \mathrm{C}$ & $T=65^{\circ} \mathrm{C}$ & $T=85^{\circ} \mathrm{C}$ & & & \\
\hline 250.0 & 215.9 & 158.1 & 105.6 & 3.27 & 2.45 & 987 \\
\hline
\end{tabular}

increasing temperature for a given humidity. Even though an increase in the water content with temperature of the membranes swollen in liquid water is clearly observed ${ }^{9,39,43}$ for the humidity range of interest in this work, the influence of temperature appears to be negligible. Thus, in this study, the $\lambda-\mathrm{RH}$ relationship is assumed to be independent of temperature. However, because we will use water volume fraction as a variable, once the effect of temperature on sorption behavior is established, this relationship can easily be incorporated into the model.

\section{YOUNG'S MODULUS OF PFSA MEMBRANES}

\section{Experimental Data on Young's Modulus of PFSA Membranes}

Experimental studies of the mechanical properties of PFSA membranes are available in the literature, where various test methods have been used to measure Young's modulus and investigate the effect of humidity and/or temperature. In various studies, uniaxial tensile testing, ${ }^{34,45,46}$ dynamical mechanical analysis (DMA), ${ }^{22,47,48}$ tapered element oscillating microbalance (TEOM), and optoelectronic holography technique $(\mathrm{OHT})^{35,42}$ have been used. These studies all show that Young's modulus decreases with water uptake (e.g., increased humidity).

Tang et al. ${ }^{34}$ conducted an experimental study to investigate the simultaneous influence of temperature and humidity on the mechanical properties of PFSA membranes and reported that Young's modulus also decreases with temperature. In that study, tensile tests for Nafion 112 membrane were conducted at four temperatures $\left(25-85{ }^{\circ} \mathrm{C}\right)$ and four humidities $(30-90 \% \mathrm{RH})$ to determine several mechanical properties, including Young's modulus. The tensile tests were conducted in two orientations, machine direction (MD) and transverse direction (TD). As similar results for the two directions were obtained, indicating a nearly isotropic in-plane behavior, the average of the values in the two directions are used in this work. The polymer volume fractions for the test samples at these $\mathrm{RH}$ values are computed using eqs 3 and 4 . The experimental data for Young's modulus of Nafion 112 membrane as a function of the reciprocal polymer volume fraction, $1 / \phi_{\mathrm{p}}$ (equal to the volume change with respect to the dry state, $V / V_{\mathrm{p}}$ ) at four temperatures is shown in Figure 4. A curve fit to the experimental data gives the following empirical relationship.

$$
E=E_{\mathrm{dry}} \phi_{\mathrm{p}}^{n}
$$

where $E_{\text {dry }}(\mathrm{MPa})$ is Young's modulus of dry Nafion 112 membrane at $\phi_{\mathrm{p}}=1,\left(\phi_{\mathrm{w}}=0\right)$ and $n$ is a power-law exponent assumed to be temperature independent. The values of $E_{\text {dry }}$ at the four temperatures, and the corresponding $n$ value are given in Table 1. The decrease in Young's modulus with increasing water content follows a similar trend at all temperatures. Moreover, we find that Young's modulus of dry PFSA membrane also decreases with increasing temperature. The relationship between $E_{\text {dry }}(\mathrm{MPa})$ and temperature (K) can be represented empirically by the following linear function:

$$
E_{\text {dry }}=E_{\text {dry }}^{0}-\kappa T,
$$

where $E_{\mathrm{dry}}^{0}(\mathrm{MPa})$ and $\kappa(\mathrm{MPa} / \mathrm{K})$ are empirical constants (Table 1). The temperature range used in this study excludes the glass transition temperature, $T_{\mathrm{g}}$. Studies pertaining to the thermal behavior of PFSA membranes report $T_{\mathrm{g}}$ values between $95-125^{\circ} \mathrm{C}$, depending on EW and hydration level. ${ }^{22,47,49}$ Thus, within the temperature range $25-85{ }^{\circ} \mathrm{C}$, it is reasonable to assume that $E_{\text {dry }}$ can be approximated by eq 7 . We will use the $E_{\text {dry }}$ values (Table 1) as an initial condition in our 
micromechanical model, and then predict how Young's modulus of the membrane changes as its water content increases at fixed temperature.

\section{Previous Models for Young's Modulus of PFSA Membranes}

Hsu and Gierke ${ }^{8,11}$ presented a semiphenomenological elastic theory for ionic clustering in PFSA membranes and proposed an expression for the cluster diameter corresponding to the minimization of the free energy. In their model, the change in the shear modulus of the matrix with change in water content is implemented empirically using a formula which can be derived theoretically from an effective medium approximation for random composites as discussed in the work of $\mathrm{Hsu}$ et al. ${ }^{50}$ This empirical formula for Young's modulus is given by, 8,11

$$
E=E_{0} \exp \left[-\alpha\left(100 \Delta m_{\mathrm{w}}+\frac{1200-\mathrm{EW}}{20}\right],\right.
$$

where $\mathrm{EW}$ is the equivalent weight, $\Delta m_{\mathrm{w}}$ is the water mass uptake of the swollen polymer per $100 \mathrm{~g}$ of dry polymer, and $E_{0}=275 \mathrm{MPa}$ and $\alpha=$ 0.0294 are empirical constants.

$\mathrm{Li}$ and Nemat-Nasser ${ }^{51}$ studied the ionic clustering in Nafion membranes and used a micromechanics approach to develop a model to calculate the effective tensile modulus of water-swollen Nafion membranes. Their studies are based on the assumption that the cluster shape is determined by the electroelastic interaction energy between the ionic clusters and the fluorocarbon matrix, and the surface energy of the cluster. Moreover, the effective Young's modulus of Nafion membranes as a function of water volume fraction is determined using a two-phase multiinclusion model for various cluster aspect ratios. They compared their findings of cluster diameter and elastic modulus with the values from literature $^{7,8,11}$ for different EW, cation forms, and water contents. The results from $\mathrm{Li}$ and Nemat-Nasser ${ }^{51}$ are reproduced graphically and shown in Figure 5 .

Li et al. ${ }^{52}$ developed a multiscale constitutive model for PFSA membranes based on the theory of poroelasticity using a two-dimensional RVE consisting of a spherical inclusion. Their results for the effective Young's modulus as a function of water volume fraction is in line with the results obtained from the empirical model of $\mathrm{Hsu}$ and Gierke. $^{11}$
The Mori-Tanaka method can also be used to estimate the effective Young's modulus of PFSA membranes in tension by assuming that the clusters are inclusions made up primarily of water. To illustrate this, we adopt a simplified version of the Mori-Tanaka method derived by Benveniste ${ }^{53}$ as applied to spherical inclusions. Using this method, the effective bulk modulus of the medium is given by

$$
K_{\text {eff }}=K_{\mathrm{m}}+\phi_{i} \frac{\left(K_{\mathrm{i}}-K_{\mathrm{m}}\right)\left(3 K_{\mathrm{m}}+4 G_{\mathrm{m}}\right)}{3 K_{\mathrm{i}}+4 G_{\mathrm{m}}-3 \phi_{\mathrm{i}}\left(K_{\mathrm{i}}-K_{\mathrm{m}}\right)},
$$

where subscripts $i$ and $m$ denote inclusions and matrix, respectively, and $\phi_{\mathrm{i}}\left(=1-\phi_{\mathrm{m}}\right)$ is the volume fraction of the inclusions. Similarly, the effective shear modulus is

$$
G_{\text {eff }}=G_{\mathrm{m}}+\frac{\phi_{i}\left(G_{\mathrm{i}}-G_{\mathrm{m}}\right)}{1+\left(1-\phi_{i}\right) \frac{6 K_{\mathrm{m}}+12 G_{\mathrm{m}}}{15 K_{\mathrm{m}}+20 G_{\mathrm{m}}}\left(\frac{G_{\mathrm{i}}-G_{\mathrm{m}}}{G_{\mathrm{m}}}\right)} .
$$

Even though water has virtually zero shear modulus $\left(G_{\mathrm{i}}=0\right)$ it has a nonzero bulk modulus. Once these effective properties are established, the effective Young's modulus can be determined from the standard relationship among material parameters for an isotropic material:

$$
E_{\text {eff }}=\frac{9 K_{\text {eff }} G_{\text {eff }}}{3 K_{\text {eff }}+G_{\text {eff }}} .
$$

We will assume that the polymer matrix is represented by dry PFSA membrane (including the ionexchange sites), and the inclusion is only water. Thus, $K_{\mathrm{m}}$ is set to the bulk modulus of dry Nafion 112 membrane, which is calculated from $E_{\text {dry }}$ (Table 1) using $K_{\mathrm{m}}=E_{\mathrm{dry}} /\left(3-6 v_{\mathrm{m}}\right)$, where $v_{\mathrm{m}}$ is Poisson's ratio of the polymer matrix. For a lack of a better measurement, we set this Poisson's ratio equal to that of $\mathrm{PTFE}^{54}$ since PTFE is the backbone of the PFSA polymer. The values of the parameters and material properties used in this model are summarized in Table 2 . The predictions for Young's modulus of Nafion 112 membrane based on $\mathrm{Li}$ and Nemat-Nasser, ${ }^{51}$ the MoriTanaka model for spherical inclusions and the empirical model shown in eq $8^{8,11}$ are depicted in Figure 5 and compared with the experimental data at $25^{\circ} \mathrm{C} .{ }^{34}$

We can see from Figure 5 that none of the existing mechanics-based models, which are all based on the assumption that the water resides only in spherical clusters, reproduce the experimental data. Therefore, we propose to include the 
Table 2. The Values of the Material Properties Used in the Calculations Given for Water and Polymer Matrix, Which is Assumed to have the Properties of a Dry Nafion Membrane

\begin{tabular}{lll}
\hline \multicolumn{1}{c}{ Material Property } & Value (Unit) & \multicolumn{1}{c}{ Notes and Comments } \\
\hline Poisson's ratio for water, $v_{\mathrm{w}}$ & 0.50 & Incompressibility \\
Poisson's ratio for polymer matrix, $v_{\mathrm{p}}$ & 0.46 & Set to the value of PTFE \\
Bulk Modulus for Water, $K_{\mathrm{w}}$ & $2250(\mathrm{MPa})$ & \\
Bulk Modulus for Polymer matrix, $K_{\mathrm{p}}$ & $1065(\mathrm{MPa})$ & Found from $E_{\text {dry }}\left(T=25^{\circ} \mathrm{C}\right)$ \\
Density of water & $1.00\left(\mathrm{~g} / \mathrm{cm}^{3}\right)$ & \\
Density of polymer matrix & $2.05\left(\mathrm{~g} / \mathrm{cm}^{3}\right)$ & Dry Nafion membrane ${ }^{36}$ \\
Equivalent weight, EW & $1100\left(\mathrm{~g} / \mathrm{mol}^{\circ}\right)$ & Nafion 112 membrane \\
\hline
\end{tabular}

channels in our micromechanics-based model, which will be discussed next.

The two-phase nanostructure of PFSA membranes seemingly exhibits structural similarities to the cellular solids, for example, the hydrophobic polymer matrix can be seen as the solid cell walls of the foam and the water domains represent the void. However, maximum water (void) volume fraction in a saturated PFSA membrane is less than 0.30, which is far lower than the porosity values required to be considered as a cellular solid. ${ }^{55}$ Not surprisingly, the predictions of the formulations of the open- and closed-cell foams for the effective modulus of PFSA membranes fail to match the experimental data (not shown for brevity). Thus, in our work, within the humidity range of interest, it is not appropriate to consider PFSA membrane as an open-cell foam structure. Nevertheless, the applicability of the cellular solid analogy could be investigated for the mechanical behavior of highly swollen or solution form of PFSA membranes, which is left to the future work due to the lack of experimental data.

\section{PROPOSED MICROMECHANICS MODEL}

\section{Representative Volume Element}

Gierke et al. $^{7}$ and $\mathrm{Hsu}$ and Gierke ${ }^{11}$ studied the clustering mechanisms and the cluster size in PFSA membranes using a simple geometry, based on the cluster-network model described earlier. In their model, sulfonic acid groups $\left(\mathrm{SO}_{3}{ }^{-}\right)$and water molecules are held in spherical clusters and channels. These clusters occupy cubic lattice sites within the polymer matrix, and are connected by cylindrical channels. Upon water absorption and consequent swelling, it is assumed that water is held only within the clusters and channels, which are surrounded by the sulfonic acid groups. As the membrane swells, the clusters and channels grow accordingly, causing growth in the volume of the cubic lattice [Fig. 2(C,D)]. The volume of the cluster plus channels is, then, the sum of the volume of the $\mathrm{SO}_{3}{ }^{-}, V_{\mathrm{SO}_{3}}$, and the absorbed water, $\Delta V_{\mathrm{w}}$. In this work, we will define the region consisting of the clusters and channels as the "pore" volume. Thus, the pore volume fraction is

$$
\phi_{\text {pore }}=\frac{V_{\text {pore }}}{V_{\mathrm{L}}}=\frac{V_{\mathrm{SO}_{3}}+\Delta V_{\mathrm{w}}}{V_{\mathrm{L}}}=\phi_{\mathrm{SO}_{3}}+\phi_{\mathrm{w}},
$$

where $V_{\mathrm{L}}$ is the total volume of the cubic lattice, and $\phi_{\mathrm{SO}_{3}}$ is the volume fraction of sulfonic acid groups and can be written as

$$
\phi_{\mathrm{SO}_{3}}=\frac{\bar{V}_{\mathrm{SO}_{3}} \operatorname{mol}\left(\mathrm{SO}_{3}^{-}\right)}{V_{\mathrm{p}} / \phi_{\mathrm{p}}}=\bar{V}_{\mathrm{SO}_{3}} \frac{\rho_{\mathrm{p}}}{\mathrm{EW}} \phi_{\mathrm{p}},
$$

where $\bar{V}_{\mathrm{SO}_{3}}$ is the molar volume of the sulfonic acid groups taken to be $40.94 \mathrm{~cm}^{3} / \mathrm{mol}$. (In that study, the approximate volume of an ion-exchange site is given as $68 \times 10^{-24} \mathrm{~cm}^{3}$ corresponding to a radius of $\sim 0.25 \mathrm{~nm}$. Here, the molar volume is obtained by multiplying by Avogadro's number, $6.02 \times 10^{23}$. . $^{7}$ Thus, $\phi_{\text {pore }}$ can be calculated as a function of the water (or polymer) volume fraction for a given EW. This calculation takes into account the expansion of the lattice during swelling, and the increase in the number of sulfonic acid groups per cluster (i.e., structural reorganization). As, by definition, the cubic lattice always contains one cluster, the number of clusters in a given volume decreases to accommodate this cluster reorganization as water is absorbed. As a result, both the total volume and the total number of $\mathrm{SO}_{3}{ }^{-}$groups in the cubic element increase, as the volume fractions of polymer matrix and $\mathrm{SO}_{3}{ }^{-}$ groups are always related by the EW of the PFSA as shown in eq 13. The corresponding volume 


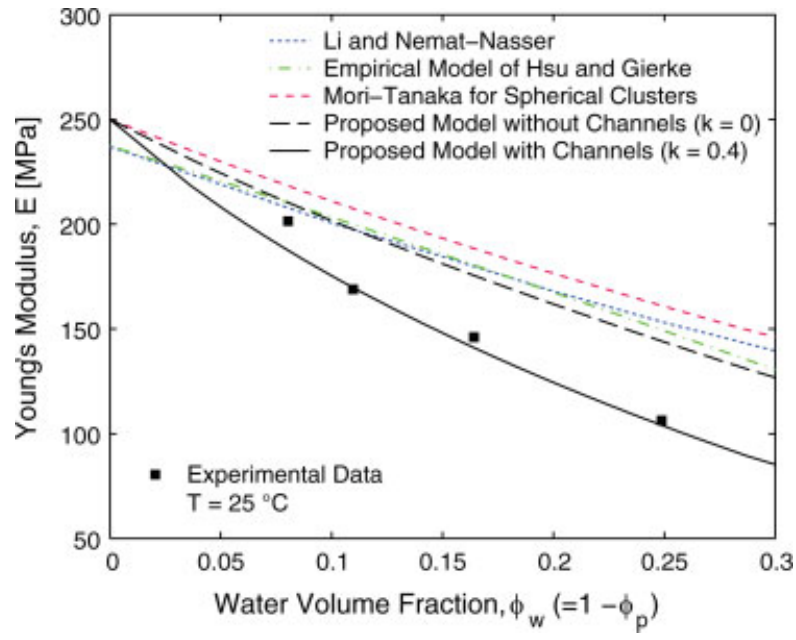

Figure 5. Young's modulus of Nafion 112 membrane at $25{ }^{\circ} \mathrm{C}$ predicted by the empirical model of $\mathrm{Hsu}$ and Gierke, ${ }^{8,11} \mathrm{Li}$ and Nemat-Nasser, ${ }^{51}$ Mori-Tanaka Model for spherical inclusions compared with the experimental data ${ }^{34}$ (markers). The proposed model is included for the RVE consisting of (i) spherical cluster only $(k=0.0)$ and (ii) spherical cluster and cylindrical channels $(k=0.4)$. [Color figure can be viewed in the online issue, which is available at www.interscience. wiley.com.]

fractions of water $\left(\phi_{\mathrm{w}}\right)$ and cluster $\left(\phi_{\text {pore }}=\phi_{\mathrm{w}}+\right.$ $\left.\phi_{\mathrm{SO}_{3}}\right)$ as a function of water content, $\lambda$, are plotted in Figure 3.

Following a similar approach to that of $\mathrm{Hsu}$ and Gierke, ${ }^{7,8,11}$ we introduce a cubic RVE of edge length $d$, to characterize the nanostructure of PFSA membranes. In this model, the cluster, where the water molecules and sulfonic acid groups are held, is represented by a spherical domain of diameter $d_{\mathrm{s}}$, in the center of the cubic volume element of the polymer matrix, connected to its four nearest neighbors by cylindrical channels of diameter, $d_{\mathrm{ch}}$ [Fig. 6(A)]. The geometry of the channels in this model is inspired by the spatial distribution of the clusters in the local-order diamond-lattice model, ${ }^{15,24,33}$ where each cluster sits in the center of a tetrahedron, with neighboring clusters at each of the four corners. Thus, our proposed RVE is a combination of the original clusternetwork model of Hsu and Gierke ${ }^{8}$ and the diamond-lattice model of Dreyfus, ${ }^{24}$ and represents a geometrically repeatable unit cell based on these models, but it is not exactly same as either of the original models. The selected geometry is chosen to obtain a simple RVE representing a homogeneous, isotropic distribution of spherical clusters.

Thus, the total volume of the water and sulfonic acid groups must be equal to the sum of the volume of the sphere and the four channels in the RVE. Previous studies suggest that the channels expand along with the spheres during water uptake. ${ }^{11,13,20,30}$ The relationship between the size of the spheres and channels is rather complicated and is determined by the energetic interactions among the water and polymer during swelling. However, for simplicity we will assume that the channel diameter increases with increasing sphere diameter. Thus, a model parameter, $k$, is introduced to represent this relationship:

$$
k=d_{\mathrm{ch}} / d_{\mathrm{s}} .
$$

When $k=0$, this model reduces to a sphere in a cubic RVE without channels. Determining the volume of a cylindrical channel under the assumed lattice structure requires some additional geometrical assumptions. In this model, we will assume that each cylindrical channel lies on the diagonal of the cubic RVE, between the surface of the sphere and the corner of the cube [Fig.
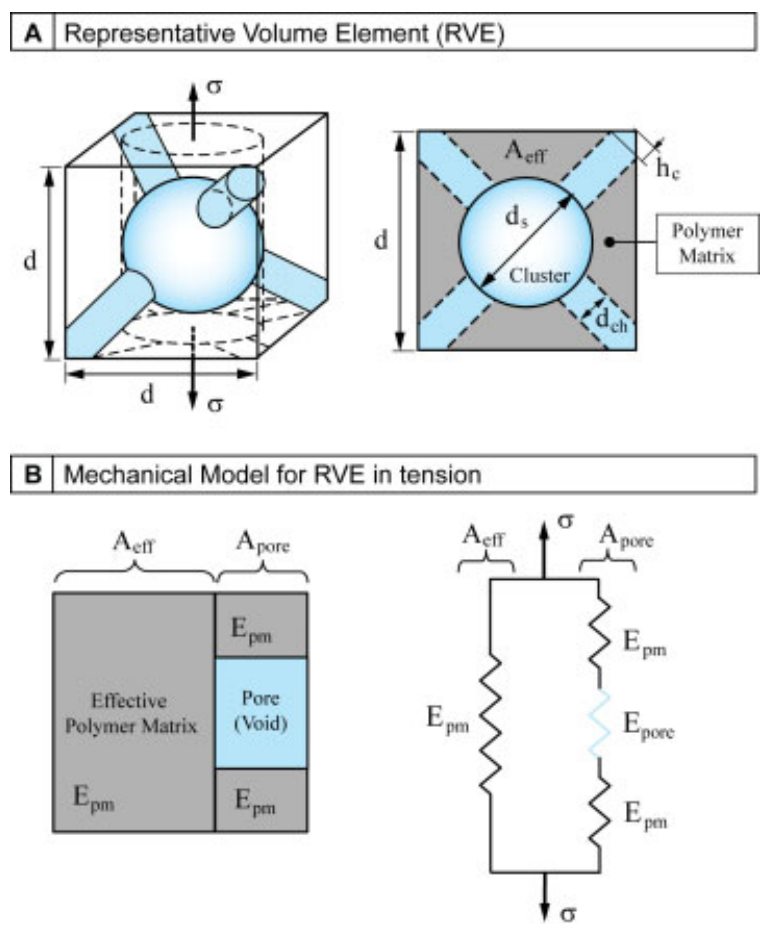

Figure 6. (A) Representative volume element (RVE) consisting of a sphere connected by four cylindrical channels subjected to a mechanical load and the projected area. (B) The corresponding mechanical model for RVE under tension, represented by linear spring elements having stiffness of $E_{\mathrm{pm}}$ for polymer matrix, and $E_{\text {pore }}$ for the pore area. [Color figure can be viewed in the online issue, which is available at www.interscience.wiley.com.] 
6(A)]. The following formula can be obtained for the volume of one channel:

$$
\left.V_{\mathrm{ch}}=\pi d_{\mathrm{ch}}^{2} \frac{d \sqrt{3}-d_{\mathrm{s}}}{2}-h_{\mathrm{c}}+\frac{1}{3} h_{\mathrm{c}}\right),(
$$

where $h_{\mathrm{c}}=\sqrt{5} \not q_{\mathrm{ch}} / 2$ is the height of a tetrahedron whose inscribed circle is the cross section of the channel area. (Once the edges of the cylinder touch the surfaces of cube, the region beyond this point can be approximated as a cone of base diameter $d_{\mathrm{ch}}$ and its height is that of a tetrahedron whose inscribed circle is the base of the cone.) The region where the surface of the sphere and the flat end of the cylinder connect is neglected. (Separate calculations show that inclusion of this region changes the resulting Young's modulus $0.3 \%$.) Thus, the total volume fraction of the water and sulfonic acid groups in the RVE, $\phi_{\text {pore }}$, including the four channels and the sphere, can be written as functions of the ratio $d_{\mathrm{s}} / d$ and the ratio of the channel diameter to the spherical cluster diameter, $k$,

$$
\begin{aligned}
\phi_{\text {pore }} & =\phi_{\mathrm{w}}+\phi_{\mathrm{SO}_{3}} \\
& =\frac{\pi}{6}\left[1-k^{2}(2 \sqrt{5} /(+3)] \frac{d_{\mathrm{s}}}{d}{ }^{3}+\frac{\sqrt{3}}{2}\left(\pi k^{2} \frac{d_{\mathrm{s}}}{d}{ }^{2} .\right.\right.
\end{aligned}
$$

This equation can be solved implicitly to obtain $d_{\mathrm{s}} / d$ for a given $k$ and pore (sphere plus channels) volume fractions, $\phi_{\text {pore }}$.

\section{Determination of Effective Young's Modulus}

A simple way to predict the effective properties of a composite material is by using the rule-of-mixtures, which assumes that each component's contribution to the effective property is proportional to its volume fraction. Here, we will use a modified rule-of-mixture for our RVE [Fig. 6(A)] to determine the effective Young's modulus as a function of water volume fraction. In particular, we will use the rule-of-mixture to establish the distribution of the load-carrying hydrophobic regions and the water absorbing hydrophilic domains in RVE. Once the water content is determined, the effective Young's modulus can be determined based on the ensuing morphological features. Because of the symmetry of this geometry, the predicted mechanical behavior will be isotropic, which is in agreement with our experimental observations. ${ }^{34}$ The behavior of the RVE subjected to tensile force is characterized by a mechanical model consisting of linear spring elements having stiffness $E_{\mathrm{pm}}$ for the polymer matrix, and $E_{\text {pore }}$ for the pore regions consisting of the cluster and channels [Fig. 6(B)]. As most of the cluster and channel volume is occupied by water, we assume that these regions act as voids in tension $\left(E_{\text {pore }}=0\right)$, so that the mechanical force is carried only by the polymer matrix. The polymer matrix consists primary of hydrophobic PTFE-like backbone and is therefore assumed not to interact with the water molecules. Consequently, plasticization of the polymer matrix is neglected in our model. Therefore, any decrease in the modulus of the membrane at a given temperature is due to the water content which only affects the size and shape of the pores (or the load carrying region). We are interested in the low-strain regime, therefore, small deformations are assumed so that the shape of the cluster and channels remain the same. The vertical central cylindrical element containing the sphere is subject to a nearly uniform stress in tension, since the sphere and the polymer matrix above and below are connected in series [Fig. 6(B)]. However, as the pore is treated as a void, it does not carry mechanical force so this cylindrical element becomes inactive, and the force is transferred only by the polymer matrix that surrounds the sphere. The volume above and below the channels is treated in the same manner. Therefore, when a force is applied to this model, the system undergoes a uniform strain and only the effective area, $A_{\text {eff }}\left(=A-A_{\text {pore }}\right)$, which is normal to the direction of applied force, is subject to stress. Thus, the effective Young's modulus of the RVE, $E_{\text {eff }}$, can be written as

$$
E_{\text {eff }}=E_{\mathrm{pm}} \frac{A_{\text {eff }}}{A}=E_{\mathrm{pm}}\left\{1-\frac{A_{\text {pore }}}{A}\right\},
$$

where $E_{\mathrm{pm}}$ is the modulus of the polymer matrix and $A_{\text {pore }} / A$ is the ratio of the pore (sphere plus channel) area to the total area of RVE in a twodimensional projected view. The projected sphere and channel area consists of a circle and four rectangles lying on the diagonals of the square [Fig. 6(A)]. Thus, the total area fraction of the sphere and channels is:

$$
\frac{A_{\text {pore }}}{A}=\left[\frac{\pi}{4}-k(2+k)\right]\left({\frac{d_{\mathrm{s}}}{d}}^{2}+(2 k \sqrt{2}) \frac{d_{\mathrm{s}}}{d} .\right.
$$

The ratio $d_{\mathrm{s}} / d$ for a given $k$ and $\phi_{\text {pore }}$ is found from eq 16. The effective Young's modulus then can be calculated by substituting eq 18 into 17 
$E_{\text {eff }}=E_{\mathrm{pm}}\left\{\left[\frac{\pi}{4}-k(2+k)\right]\left({\frac{d_{\mathrm{s}}}{d}}^{2}+(2 k \sqrt{2})\left(\frac{d_{\mathrm{s}}}{d}\right\}\right.\right.$

When the membrane is dry, $\phi_{\mathrm{p}}=1$ and the spheres and channels consist of sulfonic acid groups only, that is, $\phi_{\text {pore }}=\bar{V}_{\mathrm{SO}_{3}} \rho_{\mathrm{p}} / \mathrm{EW}$. Thus, for this case, eq 19 provides a relationship between Young's modulus of polymer matrix, $E_{\mathrm{pm}}$, and Young's modulus of the dry PFSA membrane, $E_{\text {dry }}$, which is a function of temperature only (eq 7). Therefore, if Young's modulus of the PFSA membrane is known at any water (or polymer) volume fraction, then $E_{\mathrm{pm}}$ can be determined for a given set of geometric parameters and material properties.

To use eq 19, we refer back to the literature to obtain reasonable values for the parameter $k$. On the basis of the cluster-network model, Hsu and Gierke $^{8,11}$ developed a theory for clustering and determined the cluster diameter as a function of water content. According to their calculations, the spherical cluster diameter for 1100-1200 EW PFSA polymers is within the range of $d_{\mathrm{s}}=3.6-$ $4.1 \mathrm{~nm}$ in the swollen state $(90-100 \% \mathrm{RH})$. Furthermore, both their theory and experiments show the diameter of the channels between the clusters to be $d_{\mathrm{ch}}=1.4 \pm 0.2 \mathrm{~nm}$ for $1200 \mathrm{EW}$ polymer. ${ }^{8,11}$ These values correspond to a range of $k\left(=d_{\mathrm{s}} / d_{\mathrm{ch}}\right)$ values of 0.35 to 0.45 (eq 14). In a study by Iijima et al. ${ }^{20}$ the cluster size distribution for Nafion 117 (1100 EW) membrane was determined at various water contents. On the basis of this data, they showed that the channel diameter expands during swelling assuming a cubic RVE consisting of a spherical cluster and orthotropic cylindrical channels. Even though the number of channels is higher in their model and there is scatter in the data, interpretation of their results for the swollen state gives a rough figure for $k$, which is again between 0.30 and 0.45 . Interestingly, these values are close to the total pore volume fraction at maximum humidity (Fig. 3) which might be an indication of a relationship between $k$ and the pore or water volume fraction. Thus, based on the limited available data in the literature, in our model calculations we will investigate two cases:

i. $k$ is a constant in the range of $0.35-0.45$ or,

ii. $k$ is proportional to the water volume fraction, that is, $k\left(\phi_{\mathrm{w}}\right)=k_{0} \phi_{\mathrm{w}}$, where $k_{0}$ is a constant to be determined.
Assumption ii. takes into account the percolation idea somewhat empirically, as the channels do not exist at zero water volume fraction, and the model reduces to the spherical cluster representation discussed earlier.

\section{Results for Nafion 112 Membrane}

For Nafion 112 membrane, EW is 1100 and $\rho_{\mathrm{p}}$ can be taken as $2.05 \mathrm{~g} / \mathrm{cm}^{3}$ corresponding to the density of dry Nafion membrane. ${ }^{36}$ Then, from eq 13 the corresponding volume fraction of $\mathrm{SO}_{3}{ }^{-}$groups in a dry membrane is calculated as 0.0783 . (For the other material properties used in these calculations see Table 2.) Since only $E_{\text {dry }}$ is assumed to change with temperature, by normalizing the effective Young's modulus with the modulus of the dry membrane at that temperature, that is, $\bar{E}=E_{\text {eff }} / E_{\text {dry }}$, the influence of water content on Young's modulus can be seen explicitly. The normalized effective Young's modulus determined by the proposed model is plotted in Figure 7 for various constant $k$ values and compared with the normalized experimental data. ${ }^{34}$

The nonnormalized predictions of the proposed model are compared with the experimental data ${ }^{34}$ for Young's modulus of Nafion 112 membrane as a function of water content, $\lambda$, at four temperatures $\left(25-85{ }^{\circ} \mathrm{C}\right)$ in Figure 8(A). These figures show very good agreement with the experimental data for $k$ values $0.35-0.45$. The model predictions as a function of water volume fraction are depicted in Figure 8(B) for the case $k$ increases with water volume fraction, that is, $k\left(\phi_{\mathrm{w}}\right)=k_{0} \phi_{\mathrm{w}}$ for $k_{0}$ values of 0.8 and 1.0. Higher and lower values for $k_{0}$ underpredict and overpredict the experimental data, respectively. Thus, setting $k=\phi_{\mathrm{w}}$ seems quite reasonable to reduce the number of empirical parameters and yields a very good prediction compared with the experimental data.

To confirm the validity of these $k$ values, more experimental and theoretical studies are needed. However, the fact that $k=\phi_{\mathrm{w}}$ results in a satisfactory result for Young's modulus of PFSA membranes provides a relationship between the size of the spheres and interconnecting cylindrical channels, which can be exploited in the investigation of other properties (e.g., influence of water content on the conductivity of the membrane).

When $k=0$, the channel diameters go to zero and the $\mathrm{SO}_{3}{ }^{-}$groups and water all reside in the spherical cluster. For this case, eq 19 reduces to 


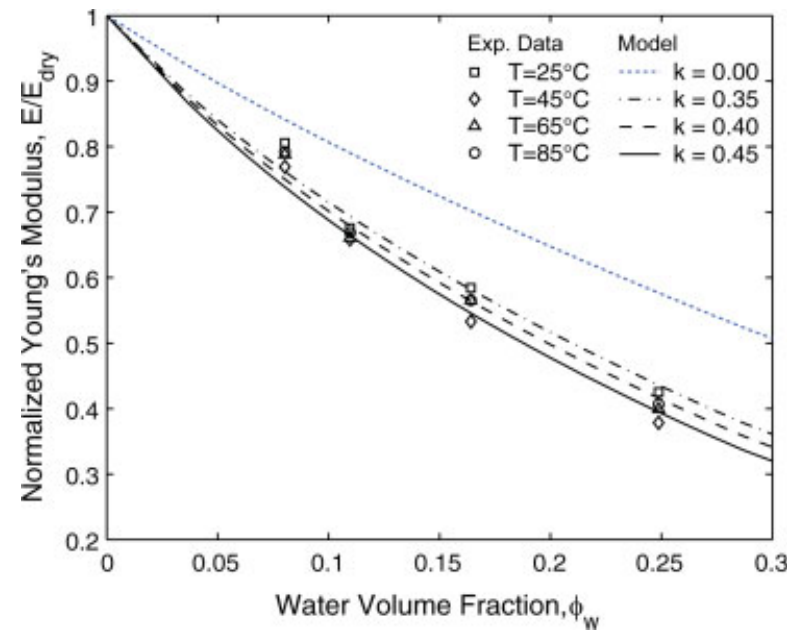

Figure 7. The normalized effective Young's moduli determined by the proposed model for various $k$ values compared with the normalized experimental data at four temperatures $\left(25-85{ }^{\circ} \mathrm{C}\right) .{ }^{34}$ [Color figure can be viewed in the online issue, which is available at www.interscience.wiley.com.]

$$
E_{\text {eff }}=E_{\mathrm{pm}}\left\{1\left(-\frac{9 \pi}{16}^{1 / 3} 1-\phi_{\mathrm{p}}+\frac{\bar{V}_{\mathrm{SO}_{3} \rho_{\mathrm{p}}}}{E W} \phi_{\mathrm{p}}\right)^{2 / 3}\right\},
$$

where $\phi_{\mathrm{p}}\left(=1-\phi_{\mathrm{w}}\right)$ is the polymer volume fraction. Now Young's modulus of the polymer matrix, $E_{\mathrm{pm}}$, can be found by setting the polymer volume fraction, $\phi_{\mathrm{p}}=1$ and $E_{\text {eff }}=E_{\text {dry }}$ in eq 20 for a given EW. Recall that $E_{\mathrm{pm}}$ is higher than $E_{\text {dry }}$ since a region consisting solely of $\mathrm{SO}_{3}$ groups exists in the dry membrane, which is excluded from the polymer matrix.

The prediction of eq 20 for Young's modulus of Nafion 112 membrane is shown in Figure 5 at 25 ${ }^{\circ} \mathrm{C}$ and compared with the previous models in the literature (see previous section) and the experimental data. ${ }^{34}$ When the RVE is represented by a spherical cluster only, and the channels are neglected, the proposed model gives similar results as the other models and consequently overpredicts Young's modulus determined from the experiments. Also, the similarity between the predictions of the proposed model without channels (eq 20) and the Mori-Tanaka model (eq 11) for the same RVE, that is, spherical inclusions, can be seen as a validation of the mechanics approach we have taken.

\section{DISCUSSION}

The proposed mechanical model uses a simple RVE to characterize the nanostructure of PFSA membranes. Considering the simplicity of our micromechanics model, the fact that we can successfully predict Young's modulus suggests a connection between the nanostructure and elastic properties of PFSA membranes. In the proposed mechanical models, we simplified the percolation phenomenon which normally requires a critical water volume fraction for the formation of channels. Instead, we investigated a case in which the channel diameter is assumed to increase with water volume fraction corresponding to an absence of channels in the dry state. Nevertheless, for PFSA membranes this critical fraction is
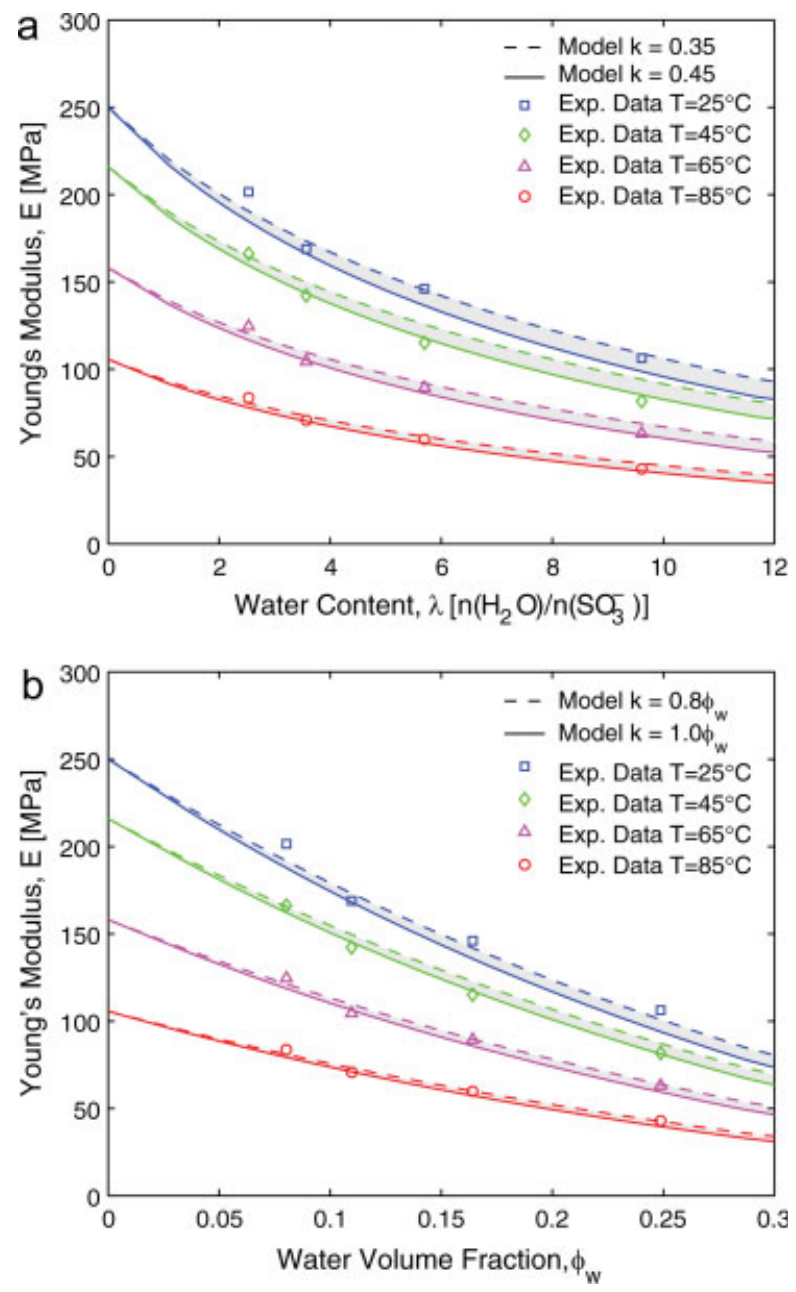

Figure 8. The predictions of the proposed model for Young's modulus of Nafion 112 membrane as a function of water content, $\lambda$, compared with the experimental data ${ }^{34}$ at four temperatures for (a) constant $k$ values of 0.35 and 0.45 , and (b) $k=0.8 \phi_{\mathrm{w}}$ and $k=$ $1.0 \phi_{\mathrm{w}}$. [Color figure can be viewed in the online issue, which is available at www.interscience.wiley. com.] 
shown to be less than $0.10 .8,30,36$ This corresponds to a transition in a very narrow range, and therefore should not significantly affect the predictions at higher water contents. The effect of percolation transition on the effective properties of polymers was investigated in the work of $\mathrm{Hsu}$ et al. ${ }^{50}$ using an effective medium theory to estimate the shear modulus assuming spherical particulates dispersed in polymer matrix. In addition, according to Gebel, ${ }^{13}$ a structural inversion occurs in PFSA membranes at around $\phi_{\mathrm{w}}=0.5$ leading to polymeric rod-like aggregates suspended in a continuous water phase. Since the water volume fractions used in this study are less than this value, any possible structural phase-transition does not invalidate the proposed RVE for the cluster-network model.

Furthermore, the thermodynamic state of the water molecules, that is, whether they are strongly bound to the sulfonic acid groups or move freely, is irrelevant within the framework of this work since only the backbone of the polymer is assumed to carry all the mechanical force. In PFSA membranes, a hydrophobic phase-separated PTFE-like backbone is observed. ${ }^{56,57}$ This observation favors our approach since, according to eq 20 , the modulus of the polymer matrix (excluding the ionic sites) is $E_{\mathrm{pm}}=320 \mathrm{MPa}$ at $T$ $=25{ }^{\circ} \mathrm{C}$, whereas the modulus of dry PFSA membrane is $E_{\text {dry }}=250 \mathrm{MPa}$. Thus, the higher stiffness of the polymer matrix can be attributed to the PTFE-like backbone. Also, the decrease in the modulus of the polymer matrix with increasing temperature (eq 7) is in agreement with the experimental observations for the modulus of PTFE. ${ }^{54}$

We considered other nanoscale representations for the structure of PFSA membranes in our mechanics-based modeling. For example, a sphere with three orthotropic channels was considered a possible RVE. However, this microstructure did not capture Young's modulus obtained experimentally. Moreover, in this case, the spheres become smaller than the cylinders as pointed out in the work of Schmidt-Rohr and Chen. ${ }^{28}$ On the other hand, if a sphere with one vertical channel is used, the model overpredicts the experimental data. The latter can be interpreted as the limiting case of the cylindrical-water channel model when the sphere and channel diameters are equal.

If the pore area fraction is calculated from eq 18 by first solving eq 16 for a range of pore volume fractions, $\phi_{\text {pore }}$, the plot of the effective area frac- tion, $A_{\text {eff }} / A$, as a function of water volume fraction, $\phi_{\mathrm{w}}$, (curve-fit to the solution) obeys an Arhenius type of formula resulting in the expression

$$
\frac{E_{\text {eff }}}{E_{\mathrm{pm}}}=\frac{A_{\text {eff }}}{A}=1-\frac{A_{\text {pore }}}{A}=B \exp \left(-\beta \phi_{\mathrm{w}}\right),
$$

where $\beta$ is a material constant that depends on the value of $k$, EW and other material properties used in the calculations. $B$ is the ratio of the dry modulus to the modulus of the polymer matrix, that is, $B=E_{\mathrm{dry}} / E_{\mathrm{pm}}$ satisfying the initial condition, $E_{\text {eff }}=E_{\text {dry }}$ when $\phi_{\mathrm{w}}=1$. Thus, eq 21 simplifies to

$$
E_{\text {eff }}=E_{\text {dry }} \exp \left(-\beta \phi_{\mathrm{w}}\right),
$$

which requires only the knowledge of the material constant, $\beta$. This formula is similar to the empirical models given in the literature ${ }^{8,11,35}$ in form but with different constants, probably due to the differences in experimental technique and the specific membrane material tested. However, our model differs from the empirical equations because the parameter $\beta$ in eq 22 is not found empirically. It is found from the solution of eqs 16-18 for a given $k$ and $\mathrm{EW}$, which depend on the RVE and the material properties, respectively. For example, for $1100 \mathrm{EW}$ membrane, $\beta$ equals to 3.31 and 3.71 for the $k$ values of 0.35 and 0.45 , respectively. Note that if $k$ changes with volume fraction, obtaining such relationship is cumbersome. but we found that eq 22 can still be used as a first-order approximation.

Young's modulus of PFSA membranes subjected to compression is of great importance, since these membranes are subject to significant compressive loads when used in fuel cells. ${ }^{6}$ However, to date no experimental data has been reported on Young's modulus of PFSA membranes in compression. Modeling the compressive behavior may require a different treatment since water behaves differently in compression than tension. The nanostructure-property relationship in PFSA membranes must be explored further, considering other morphological models proposed in the literature. For example, swelling and mechanical deformation of the membrane might induce microstructural changes in the orientation and shape of the clusters, requiring more sophisticated models to take into account these affects. Finally, more experimental data is needed for the validation of the model at higher water contents and for different membranes. 


\section{CONCLUSIONS}

In this study, a micromechanics-based model is developed to determine Young's modulus of PFSA membranes as a function of water content at various temperatures. Based on the cluster-network model, the nanostructure of PFSA membrane is characterized by a RVE: water filled pores consisting of a sphere connected by four cylindrical channels in a cubic polymer matrix. The swelling of the membrane is modeled by the growth of the clusters and the resulting growth in the RVEs. The mechanical load is carried by the polymer matrix consisting of hydrophobic PTFE-like backbone. The properties of polymer matrix are related to that of dry PFSA membrane, which are affected by the temperature only. Water content determines only the shape and size of the pores and, therefore, the associated decrease in the modulus of membrane at a given temperature. By performing a simple mechanics analysis for the RVE, a theoretical formula is proposed for Young's modulus of PFSA membranes. The significance of this work stems from the fact that we have eliminated the need for any empirical constants, and the material parameters in the proposed model all have clear physical meanings, which are attributed to the geometry of the clusters and channels in the RVE. When the cluster is represented by a sphere connected by four channels, the proposed model demonstrates very good agreement with the experimental data for the effective Young's moduli of Nafion 112 membranes in the temperature range of $25-85{ }^{\circ} \mathrm{C}$ and water volume fractions up to 0.3 . The RVE simplifies to spherical inclusions in the absence of channels. In this case, the predictions of the model are similar to those of other semiempirical models in the literature and the Mori-Tanaka model for spherical inclusions, which all significantly overpredict Young's modulus compared with the experimental data. Thus, these results suggest that there is a strong connection between the mechanical properties and the nanostructure of PFSA membranes. We were able to show that when an accurate representation of the nanostructure is used, the micromechanics model agrees well with the experimental results. The model presented in this study can be used to accurately predict Young's modulus of PFSA membranes without the need for any empirical constants.

This research has been supported by grants from W.L. Gore and Associates, Inc., the State of Delaware Devel- opment Office (DEDO), and the U.S. Department of Energy.

\section{REFERENCES AND NOTES}

1. Liu, W.; Ruth, K.; Rusch, G. J New Mater Electrochem Syst 2001, 4, 227-232.

2. Xie, J.; Wood, D. L., III; More, K. L.; Atanassov, P.; Borup, R. L. J Electrochem Soc 2005, 152, 1011-1020.

3. Kundu, S.; Fowler, M. W.; Simon, L. C.; Grot, S. J Power Sources 2006, 157, 650-656.

4. Tang, Y.; Santare, M. H.; Karlsson, A. M.; Cleghorn, S.; Johnson, W. B. J Fuel Cell Sci Technol 2006, 3, 119-124.

5. Kusoglu, A.; Karlsson, A. M.; Santare, M. H.; Cleghorn, S.; Johnson, W. B. J Power Sources 2006, 161, 987-996.

6. Kusoglu, A.; Karlsson, A. M.; Santare, M. H.; Cleghorn, S.; Johnson, W. B. J Power Sources 2007, 170, 345-358.

7. Gierke, T. D.; Munn, G. E.; Wilson, F. C. J Polym Sci: Polym Phys Ed 1981, 19, 1687-1704.

8. Hsu, W. Y.; Gierke, T. D. J Membrane Sci 1983, 13, 307-326.

9. Zawodzinski, T. A.; Springer, T. E.; Uribe, F.; Gottesfeld, S. Solid State Ionics 1993, 60, 199-211.

10. Laporta, M.; Pegoraro, M.; Zanderighi, L. Phys Chem Chem Phys 1999, 1, 4619-4628.

11. Hsu, W. Y.; Gierke, T. D. Macromolecules 1982, $15,101-105$.

12. Eisenberg, A. Macromolecules 1970, 3, 147-154.

13. Gebel, G. Polymer 2000, 41, 5829-5838.

14. Orfino, F. P.; Holdcroft, S. J New Mater Electrochem Syst 2000, 3, 285-290.

15. Dreyfus, B.; Gebel, G.; Aldebert, P.; Pineri, M.; Escoubes, M.; Thomas, M. J De Phys 1990, 51, 1341-1354.

16. Meresi, G.; Wang, Y.; Bandis, A.; Inglefield, P. T.; Jones, A. A.; Wen, W. Y. Polymer 2001, 42, 6153-6160.

17. Gebel, G.; Lambard, J. Macromolecules 1997, 30, 7914-7920.

18. Kim, M. H.; Glinka, C. J.; Grot, S. A.; Grot, W. G. Macromolecules 2006, 39, 4775-4787.

19. James, P. J; Elliott, J A.; McMaster, T. J; Newton, J M.; Elliott, A. M. S.; Hanna, S.; Miles, M. J. J Mater Sci 2000, 35, 5111-5119.

20. Iijima, M.; Sasaki, Y.; Osada, T.; Miyamoto, K.; Nagai, M. Int J Thermophys 2006, 27, 1792-1802.

21. Gebel, G.; Diat, O. Fuel Cells 2005, 5, 261-276.

22. Yeo, S. C.; Eisenberg, A. J Appl Polym Sci 1977, $21,875-898$.

23. Fujimura, M.; Hashimoto, T.; Kawai, H. Macromolecules 1982, 15, 136-144.

24. Dreyfus, B. Macromolecules 1985, 18, 284-292.

25. van der Heijden, P. C.; Rubatat, L.; Diat, O. Macromolecules 2004, 37, 5327-5336. 
26. Termonia, Y. Polymer 2007, 48, 1435-1440.

27. Mauritz, K. A.; Moore, R. B. Chem Rev 2004, 104, 4535-4585.

28. Schmidt-Rohr, K.; Chen, Q. Nat Mater 2008, 7, 75-83.

29. Eikerling, M.; Kornyshev, A. A.; Stimming, U. J Phys Chem B 1997, 101, 10807-10820.

30. Weber, A. Z.; Newman, J. J Electrochem Soc 2004, 151, 311-325.

31. Koter, S. J Membrane Sci 2000, 166, 127-135.

32. Divisek, J; Eikerling, M.; Mazin, V.; Schmitz, H.; Stimming, U.; Volfkovich, Y. M. J Electrochem Soc 1998, 145, 2677-2683.

33. Dreyfus, B. In Structure and Properties of Ionomers, NATO ASI Series C-198; Pineri, M.; Eisenberg, A., Eds.; Reidel: Dordrecht, 1987, pp 27-37.

34. Tang, Y.; Karlsson, A. M.; Santare, M. H.; Gilbert, M.; Cleghorn, S.; Johnson, W. B. Mater Sci Eng A 2006, 425, 297-304.

35. Choi, P.; Jalani, N. H.; Datta, R. J Electrochem Soc 2005, 152, E84-E89.

36. Morris, D. R.; Sun, X. D. J Appl Polym Sci 1993, 50, 1445-1452.

37. Thampan, T.; Malhotra, S.; Tang, H.; Datta, R. J Electrochem Soc 2000, 147, 3242-3250.

38. Springer, T. E.; Zawodzinski, T. A.; Gottesfeld, S. J Electrochem Soc 1991, 138, 2334-2342.

39. Onishi, L. M.; Prausnitz, J. M.; Newman, J. J Phys Chem B 2007, 111, 10166-10173.

40. Schroeder, P. Z. Phys Chem 1903, 45, 75.

41. Choi, P. H.; Datta, R. J Electrochem Soc 2003, 150, E601-E607.

42. Jalani, N. H.; Choi, P.; Datta, R. J Membr Sci 2005, 254, 31-38.
43. Hinatsu, J. T.; Mizuhata, M.; Takenaka, H. J Electrochem Soc 1994, 141, 1493-1498.

44. Takata, H.; Mizuno, N.; Nishikawa, M.; Fukada, S.; Yoshitake, M. Int J Hydrogen Energy 2007, 32, 371-379.

45. Liu, D.; Kyriakides, S.; Case, S. W.; Lesko, J. J.; Li, Y. X.; McGrath, J. E. J Polym Sci Part B: Polym Phys 2006, 44, 1453-1465.

46. Solasi, R.; Zou, Y.; Huang, X.; Reifsnider, K.; Condit, D. J Power Sources 2007, 167, 366-377.

47. Bauer, F.; Denneler, S.; Willert-Porada, M. J Polym Sci Part B: Polym Phys 2005, 43, 786-795.

48. Kundu, S.; Simon, L. C.; Fowler, M.; Grot, S. Polymer 2005, 46, 11707-11715.

49. de Almeida, S. H.; Kawano, Y. J Therm Anal Calorimetry 1999, 58, 569-577.

50. Hsu, W. Y.; Giri, M. R.; Ikeda, R. M. Macromolecules 1982, 15, 1210-1212.

51. Li, J. Y.; Nemat-Nasser, S. Mech Mater 2000, 32, 303-314.

52. Li, H.; Yuan, Z.; Ng, T. Y.; Lee, H. P.; Lam, K. Y.; Wang, Q. X.; Wu, S. N.; Fu, J.; Hanes, J. J Biomater Sci Polym Ed 2003, 14, 1181-1196.

53. Benveniste, Y. Mech Res Commun 1986, 13, 193201.

54. Rae, P. J.; Brown, E. N. Polymer 2005, 46, 81288140 .

55. Gibson, L. J.; Ashby, M. F. Cellular Solids: Structure and Properties; Cambridge University Press: New York, 1997.

56. Gruger, A.; Regis, A.; Schmatko, T.; Colomban, P. Vibration Spectrosc 2001, 26, 215-225.

57. Starkweather, H. W. Macromolecules 1982, 15, 320-323. 